5. Hijnen D, Marzano A, Eyerich K, et al. SARS-CoV-2 transmission from presymptomatic meeting attendee, Germany. Emerg Infect Dis 2020;26:1935-1937.

6. Clinical questions about COVID-19: questions and answers. Centers for Disease Control and Prevention website. https:/www.cdc.gov/coronavirus/ 2019-ncov/hcp/faq.html. Published 2020. Accessed August 25, 2020.

7. VM Dato, Hostler D, Hahn ME, Simple respiratory mask. Emerg Infect Dis 2006;12:1033-1034.

8. Uematsu J, Sakai SK, Kihira NS, et al. Inhibitions of human parainfluenza virus type 2 replication by ribavirin and mycophenolate mofetil are restored by guanosine and S-(4-nitrobenzyl)-6-thioinosine. Drug Discov Ther 2019;13:314-321.

9. Bullard J, Dust K, Funk D, et al. Predicting infectious SARS-CoV-2 from diagnostic samples. Clin Infect Dis 2020 [E-pub ahead of print]. doi: 10 . 1093/cid/ciaa638.

10. Huang C, Wang Y, Li X, Ren L, Zhao J, Hu Y, et al. Clinical features of patients infected with 2019 novel coronavirus in Wuhan, China. Lancet 2020;395:497-506.

\title{
Characterizing coinfection in children with COVID-19: A dual center retrospective analysis
}

\author{
David D. Zhang $M D^{1}{ }^{10}$, Mary Ellen Acree $M D^{2}$, Jessica P. Ridgway $M^{3}$, Nirav Shah MD, $M P^{2}$, Aniruddha Hazra MD $^{3}$, \\ Urmila Ravichandran $\mathrm{MS}^{2}$ and Madan Kumar $\mathrm{DO}^{1}$ \\ ${ }^{1}$ Department of Pediatrics, Section of Pediatric Infectious Disease, University of Chicago Medicine, Chicago, Illinois, ${ }^{2}$ Division of Infectious Disease, NorthShore \\ University HealthSystem, Evanston, Illinois and ${ }^{3}$ Department of Medicine, Section of Infectious Disease and Global Health, University of Chicago Medicine, \\ Chicago, IL
}

To the Editor-Understanding the prevalence of coinfections with coronavirus disease 2019 (COVID-19) is crucial to delineating its true clinical impact. Numerous studies have evaluated coinfections in adults with COVID-19, ${ }^{1-3}$ but data on pediatric COVID-19 coinfections are limited. Here, we evaluate the burden of coinfections in pediatric COVID-19 patients at 2 large Chicagoland medical centers.

\section{Methods}

We retrospectively reviewed electronic health records of all pediatric patients tested for severe acute respiratory coronavirus virus 2 (SARS-CoV-2) from March 9, 2020, through April 30, 2020, in 2 Chicagoland medical centers. At the University of Chicago Medicine, SARS-CoV-2 was diagnosed using one of the following real-time reverse transcriptase polymerase chain reaction (RT-PCR) assays on respiratory specimens: Cobas SARS-CoV-2 RT-PCR assay (Roche Basel, Switzerland) or Xpert Xpress SARS-CoV-2 test (Cepheid, Sunnyvale, CA). Respiratory coinfections were primarily identified using a multiplex RT-PCR respiratory viral panel (RVP) with the following targets: adenovirus, coronavirus 229E/HKU1/NL63/OC43, human metapneumovirus, influenza-A/-B, parainfluenzas 1-4, respiratory syncytial virus, Mycoplasma pneumoniae, Chlamydophila pneumoniae, Bordetella pertussis, and rhinovirus/enterovirus (FilmArray Respiratory Panel, BioFire Diagnostics, Salt Lake City, UT). Coinfections were also identified using the influenza/respiratory syncytial virus (RSV) RT-PCR assay (Cepheid Xpert Xpress Flu/RSV) known as

Author for correspondence: David D. Zhang, MD, Pediatric Infectious Disease, University of Chicago Medicine, 5837 S. Maryland Ave, MC 6054, Chicago, IL 60637. E-mail: David.zhang2@uchospitals.edu

Cite this article: Zhang DD, et al. (2021). Characterizing coinfection in children with COVID-19: A dual center retrospective analysis. Infection Control \& Hospital Epidemiology, 42: 1160-1162, https://doi.org/10.1017/ice.2020.1221 the influenza/RSV panel (IRP). At NorthShore University HealthSystem, SARS-CoV-2 was identified similarly using RT-PCR: Xpert Xpress or BD Max (Becton Dickinson, Franklin Lakes, NJ). Coinfections were detected using a multiplex RT-PCR panel that contained only the viral targets of the RVP (GenMark Dx, GenMark Diagnostics, Carlsbad, CA), as well as an IRP (Roche Cobas Liat Influenza $\mathrm{A} / \mathrm{B}$ and RSV). We included all RVPs and IRPs that were obtained within 7 days of a SARS-CoV-2 test.

We also reviewed antibiotic prescriptions within 7 days of a positive SARS-CoV-2 RT-PCR result, and we evaluated antibiotic indication to confirm whether bacterial coinfection was present. Combined means, frequencies, and standard deviations were calculated from the 2 subgroups of data. The Fisher exact test was used to detect any significant differences in proportions of coinfection between the SARS-CoV-2 positive and negative groups. Statistical significance was defined as $P<.05$. Stata version 16 software (StataCorp, College Station, TX) was used for all analyses.

\section{Results}

During the study period, 3,669 specimens were sent for SARS-CoV-2 testing, and 862 of these $(23.4 \%)$ were positive. Furthermore, 767 (20.9\%) specimens had a paired RVP or IRP within 7 days. Of these paired specimens, 101 (13.2\%) were positive for SARS-CoV-2. The average ages of our aggregate pediatric COVID cohort and the coinfected subgroup were 17.1 years (standard deviation 5.79) and 17 years (standard deviation, 5.11), respectively.

Analyses between RSV/influenza and the rest of the respiratory viral pathogen panel were conducted separately given the large number of IRPs performed (ie, 351 total multiplex RVPs and 424 total $\mathrm{RSV} /$ influenza IRPs). Only 2 paired specimens containing the RVP (12.5\%) tested positive for SARS-CoV-2 and an additional respiratory pathogen (Table 1). In 1 of these 2 patients, 2 viral pathogens on the RVP (rhino/enterovirus and adenovirus) were detected. Of those who tested negative for SARS-CoV-2 who had the RVP, 130 (38.8\%) tested

(C) 2020 by The Society for Healthcare Epidemiology of America. All rights reserved. This is an Open Access article, distributed under the terms of the Creative Commons Attribution licence (http://creativecommons.org/licenses/by/4.0/), which permits unrestricted re-use, distribution, and reproduction in any medium, provided the original work is properly cited. 
Table 1. Proportions of Positive Respiratory Viral Pathogens Stratified by SARS-CoV-2

\begin{tabular}{|c|c|c|c|}
\hline Variable & SARS-CoV-2 Positive, no. (\%) & SARS-CoV-2 Negative, no. (\%) & $P$ Value $^{b}$ \\
\hline Patients undergoing multiplex RVP testing ${ }^{a}$ & $(n=16)$ & $(n=335)$ & \\
\hline Positive for any respiratory pathogen & $2(12.5)$ & $130(38.8)$ & .036 \\
\hline Adenovirus & $1(6.3)$ & $24(7.2)$ & \\
\hline Coronavirus 229E & $0(0.0)$ & $2(0.6)$ & \\
\hline Coronavirus HKU1 & $0(0.0)$ & $2(0.6)$ & \\
\hline Coronavirus NL63 & $0(0.0)$ & $11(3.3)$ & \\
\hline Coronavirus OC43 & $0(0.0)$ & $5(1.5)$ & \\
\hline Human metapneumovirus & $0(0.0)$ & $25(7.5)$ & \\
\hline Parainfluenza 1 & $0(0.0)$ & $2(0.6)$ & \\
\hline Parainfluenza 2 & $0(0.0)$ & $2(0.6)$ & \\
\hline Parainfluenza 3 & $0(0.0)$ & $1(0.3)$ & \\
\hline Parainfluenza 4 & $0(0.0)$ & $3(0.9)$ & \\
\hline Mycoplasma pneumoniae & $0(0.0)$ & $0(0.0)$ & \\
\hline Chlamydophila pneumoniae & $0(0.0)$ & $0(0.0)$ & \\
\hline Bordetella pertussis & $0(0.0)$ & $1(0.3)^{c}$ & \\
\hline Rhino/enterovirus & $2(12.5)$ & $78(23.3)$ & \\
\hline Patients tested for RSV and influenza & $(n=101)$ & $(n=666)$ & \\
\hline Positive for RSV or influenza & $2(2.0)$ & $39(5.9)$ & .151 \\
\hline Influenza A & $1(1.0)$ & $4(0.6)$ & \\
\hline Influenza B & $0(0.0)$ & $12(1.8)$ & \\
\hline Respiratory syncytial virus & $1(1.0)$ & $25(3.8)$ & \\
\hline
\end{tabular}

Note. RSV, respiratory syncytial virus; RVP, respiratory viral panel.

aRSV and influenza analyzed separately given large volume of RSV/influenza (IRP) tests.

${ }^{\text {b }}$ Statistical significance determined by the Fisher exact test. Every individually stratified respiratory pathogen had $P>.05$.

'Bacterial targets were not included in a minority of multiplex RT-PCR tests performed on GenMark Dx. The denominator for these targets is 323.

positive for at least 1 pathogen, excluding RSV and influenza. The most common pathogen isolated was rhino/enterovirus $(23.3 \%)$. Specimens positive for SARS-CoV-2 with a paired RVP were significantly less likely to be positive for any other respiratory pathogen than specimens that were negative for SARS-CoV-2 $(P=.036)$. Only 2 (2.0\%) paired specimens tested positive for both SARS-CoV-2 and either RSV or influenza, whereas 39 paired specimens $(5.9 \%)$ that were positive for either RSV or influenza tested negative for SARS-CoV-2 (Table 1). No association was found between individual respiratory pathogens and SARS-CoV-2 status.

Among all pediatric patients who tested positive for SARS-CoV-2, without respect to having a paired RVP/IRP, there were 35 patients (4\%) who received antibiotics within 7 days before and after the SARS-CoV-2 assay. Among these patients, only 8 of 35 COVID-19 patients $(23 \%)$ receiving antibiotics had an indication of lower respiratory tract infection (LRTI), and streptococcal pharyngitis (12 of 35, $34 \%$ ) was the most common non-LRTI indication.

\section{Discussion}

Recent reports have shown pediatric COVID-19 coinfection rates as high as $51 \%{ }^{4,5}$ However, our dual-center study revealed that viral coinfection rates in pediatric COVID-19 patients are low. This analysis was performed at a time of year when respiratory viral transmission, most notably influenza, was declining.
During the study period, the Illinois Department of Public Health tracked a decrease in positive influenza tests from $14.9 \%$ for the week ending March 14, 2020, to $1.8 \%$ for the week ending April 25, 2020. ${ }^{6}$ The stay-at-home order issued in the state of Illinois on March 9, 2020, also may have played a role in the reduction of seasonal respiratory viral transmission. ${ }^{7}$ During seasons of low rates of respiratory viral transmission, our data suggest that testing for other viruses among COVID-19 patients may not be warranted.

A recent review suggested that antibiotic usage in COVID-19 patients reached $72 \% .^{8}$ Antibiotic prescription rates in our pediatric cohort were much lower, suggesting that bacterial coinfection is less likely in pediatric COVID-19 patients. Additionally, antibiotic indications were rarely for lower respiratory tract infection. The most common indication was streptococcal pharyngitis, which may have been incidental.

The limitations of this study include the lack of stratification between inpatients and outpatients, as well as stratification between different age groups because both may impact coinfection rates.

Our findings suggest that viral coinfections in pediatric patients with SARS-CoV-2 likely correlate with general respiratory infection rates. However, more longitudinal studies that span the entire viral respiratory season are needed to clarify the rate of secondary infections in this population. 


\section{Acknowledgments.}

Financial support. No financial support was provided relevant to this article.

Conflicts of interest. All authors report no conflicts of interest relevant to this article.

\section{References}

1. Hazra A, Collison M, Pisano J, et al. Coinfections with SARS-CoV-2 and other respiratory pathogens. Infect Control Hosp Epidemiol 2020 Jul 3. doi: 10.1017/ice.2020.322.

2. Kim D, Quinn J, Pinsky B, Shah NH, Brown I. Rates of coinfection between SARS-CoV-2 and other respiratory pathogens. JAMA 2020;323:2085-2086.

3. Lansbury L, Lim B, Baskaran V, Lim WS. Coinfections in people with COVID-19: a systematic review and meta-analysis. J Infect 2020 May 27. doi: 10.1016/j.jinf.2020.05.046.

4. Wu Q, Xing Y, Shi L, et al. Coinfection and other clinical characteristics of COVID-19 in children. Pediatrics 2020;146(1):e20200961.
5. Mannheim J, Gretsch S, Layden JE, Fricchione MJ. Characteristics of hospitalized pediatric COVID-19 cases-Chicago, Illinois, March-April 2020. J Pediatric Infect Dis Soc 2020 Jun 1 [Epub ahead of print]. doi: 10.1093/ jpids/piaa070.

6. Influenza surveillance: 2019-2020 flu activity report. Illinois Department of Public Health website. https://dph.illinois.gov/topics-services/diseases-andconditions/influenza/influenza-surveillance. Published April 25, 2020. Accessed July 13, 2020.

7. Weisenstein B. Pritzker gives stay-at-home order for Illinois, in effect until April 7. Illinois Policy website. https://www.illinoispolicy.org/pritzkerorders-illinoisans-to-shelter-in-place-until-april-7. Published March 20, 2020. Accessed July 13, 2020.

8. Rawson TM, Moore LSP, Zhu N, et al. Bacterial and fungal coinfection in individuals with coronavirus: a rapid review to support COVID-19 antimicrobial prescribing. Clin Infect Dis 2020. May 2 [Epub ahead of print]. doi: 10. 1093/cid/ciaa530.

\title{
Construction of a container isolation ward: A rapidly scalable modular approach to expand isolation capacity during the coronavirus disease 2019 (COVID-19) pandemic
}

\author{
Liang En Wee MRCP ${ }^{1,2}$ (1), Esther Monica Peijin Fan $\mathrm{BSc}^{3}$, Raphael Heng B.Eng ${ }^{4}$, Shin Yuh Ang MBA ${ }^{3}$, \\ Juat Lan Chiang MHSc(Mgt) ${ }^{3,4}$, Thuan Tong Tan PhD², Moi Lin Ling FRCPA ${ }^{5}$ and Limin Wijaya MRCP 2 \\ ${ }^{1}$ SingHealth Infectious Diseases Residency, Singapore, ${ }^{2}$ Department of Infectious Diseases, Singapore General Hospital, Singapore, ${ }^{3}$ Division of Nursing, \\ Singapore General Hospital, Singapore, ${ }^{4}$ Facilities and Infrastructure Development, Singapore General Hospital, Singapore and ${ }^{5}$ Department of Infection \\ Prevention and Epidemiology, Singapore General Hospital, Singapore
}

To the Editor-During the ongoing coronavirus disease 2019 (COVID-19) pandemic, airborne infection isolation rooms (AIIRs) are in high demand. Surge capacity is urgently required during significant ongoing community transmission. ${ }^{1}$ Converting hospital wards to AIIRs can serve as a temporary solution, ${ }^{1,2}$ but it comes at the expense of existing capacity. Temporary tent-based structures or conversion of nonmedical facilities has been commonly proposed. ${ }^{3-5}$ Such solutions, though, may not be durable, and retrofitting nonmedical facilities to meet medical standards is difficult. Building more permanent structures with AIIR capabilities, however, is time-consuming and costly. ${ }^{5}$ We describe our institution's experience with constructing an isolation ward from prefabricated containers.

In Singapore, at the end of July 2020, >50,000 cases of COVID-19 had been reported locally. ${ }^{6}$ Our institution's purpose-built 51-bed isolation ward housed high-risk COVID-19 suspects and confirmed COVID-19 cases in AIIRs. From February 2020, given ongoing community transmission, lower-risk individuals with respiratory syndromes were housed in converted general wards, with partitions and reduced beds per cubicle to mitigate the risk of healthcare-associated transmission. ${ }^{7}$ To date, this

\section{Author for correspondence: Wee Liang En Ian, E-mail: ian.wee@mohh.com.sg}

Cite this article: Wee LE, et al. (2021). Construction of a container isolation ward: A rapidly scalable modular approach to expand isolation capacity during the coronavirus disease 2019 (COVID-19) pandemic. Infection Control \& Hospital Epidemiology, 42: 1162-1164, https://doi.org/10.1017/ice.2020.1222 containment strategy has been successful, with no cases of healthcare-associated transmission between patients and healthcare workers (HCWs). ${ }^{89}$ However, this placed pressure on bed capacity, with almost $20 \%$ of bed capacity set aside to house suspected and confirmed cases of COVID-19. Our institution thus sought to relieve the pressure by constructing an isolation ward extension.

From mid-April 2020, our institution started planning the isolation-ward extension, soon after a surge in cases (Fig. 1a). An open-air car park $\left(3,200 \mathrm{~m}^{2}\right)$ was utilized. The novel feature was the utilization of a scalable modular design using prefabricated containers. Each prefabricated container $(6 \mathrm{~m} \times 2.4 \mathrm{~m})$ was redesigned as a single-occupancy room with an en suite bathroom (Fig. 1b, c, and d). All patient rooms met design standards for AIIRs, including $\geq 12$ air changes per hour and controlled direction of air flow with a negative differential pressure of -2.5 Pascal. Each container was also equipped with oxygen, air conditioning, exhaust ventilation, and a high-efficiency particulate air (HEPA) filter. Modification of containers was done off site. One container was also customized with lead shielding and a patient booth to allow $\mathrm{X}$-rays to be performed.

At the end of May 2020, construction began (Fig. 1e). Modular construction allowed rapid construction of a 50-bed isolation ward in just 50 days, using only 100 workers, despite significant challenges. Outbreaks in the dormitories of foreign workers significantly limited manpower availability, and construction work was curtailed during a 2-month-long shutdown as part of

(c) 2020 by The Society for Healthcare Epidemiology of America. All rights reserved. This is an Open Access article, distributed under the terms of the Creative Commons Attribution licence (http://creativecommons.org/licenses/by/4.0/), which permits unrestricted re-use, distribution, and reproduction in any medium, provided the original work is properly cited. 